\title{
Papilomatosis traqueobronquial y pulmonar sin compromiso de la laringe tratada con bevacizumab endovenoso en un niño
} Tracheobronchial and pulmonary papillomatosis without involvement of the larynx treated with intravenous Bevacizumab in a child

\author{
Dra. Giselle Cuestas ${ }^{a}$, Dra. Verónica Rodrígueza , Dra. Flavia Doormann ${ }^{a}$ Dr. Patricio Bellia Munzón ${ }^{a}$ y \\ Dr. Gastón Bellia Munzón ${ }^{b}$
}

\begin{abstract}
RESUMEN
La papilomatosis respiratoria recurrente es una neoplasia benigna infrecuente que, comúnmente, afecta la vía aérea superior con predilección por la laringe. El compromiso traqueobronquial aislado es muy raro.

El diagnóstico y el tratamiento de esta enfermedad constituyen un desafío, debido a su manifestación clínica inespecífica y su naturaleza recurrente.

Se presenta a un varón de 6 años con diagnóstico de asma refractario al tratamiento, sin historia ni evidencia de papilomatosis laríngea. El examen endoscópico reveló papilomatosis traqueobronquial extensa, y la tomografía computada, compromiso pulmonar. Recibió terapia adyuvante con bevacizumab endovenoso, con muy buena respuesta.

Se alerta a los pediatras para considerar esta rara neoplasia traqueobronquial en todo niño con síntomas de asma atípicos o que no mejoran con el tratamiento médico convencional. Palabras clave: papilomatosis respiratoria recurrente, bevacizumab sistémico, niño.
\end{abstract}

\begin{abstract}
Recurrent respiratory papillomatosis is an infrequent benign neoplasm that commonly affects the upper airway with a predilection for the larynx. Isolated tracheobronchial involvement is very rare.

Diagnosis and treatment of this disease is a challenge due to its non-specific clinical manifestation and its recurrent nature. We present a 6-year-old male with a diagnosis of asthma refractory to treatment, without history or evidence of laryngeal papillomatosis. The endoscopic examination revealed extensive tracheobronchial papillomatosis and the computed tomography, pulmonary involvement. He received adjuvant therapy with intravenous Bevacizumab with very good response.
\end{abstract}

a. Sección de Endoscopía Respiratoria, División de Otorrinolaringología, Hospital General de Niños "Dr. Pedro de Elizalde", Ciudad de Buenos Aires, Argentina.

b. Servicio de Cirugía, Hospital General de Niños

"Dr. Pedro de Elizalde", Ciudad de Buenos Aires, Argentina.

Correspondencia:

Dra. Giselle Cuestas: giselle_cuestas@yahoo.com.ar

Financiamiento: Ninguno.

Conflicto de intereses: Ninguno que declarar.

Recibido: 1-7-2018

Aceptado: 4-10-2018
We alert pediatricians to consider this rare tracheobronchial neoplasm in all children with atypical asthma symptoms or in those who do notimprove with conventional medical treatment. Key words: recurrent respiratory papillomatosis, systemic Bevacizumab, child.

http: / / dx.doi.org/10.5546/ aap.2019.e72

Cómo citar: Cuestas G, Rodríguez V, Doormann F, Bellia Munzón P, et al. Papilomatosis traqueobronquial y pulmonar sin compromiso de la laringe tratada con bevacizumab endovenoso en un niño. Arch Argent Pediatr 2019;117(1):e72-e76.

\section{INTRODUCCIÓN}

La papilomatosis respiratoria recurrente (PRR) es una patología poco frecuente causada por el virus del papiloma humano (VPH), principalmente, los tipos 6 y 11, y tiene predilección por la laringe. ${ }^{1}$ El compromiso de la tráquea y de los bronquios, presente en el $8 \%$ y el $3 \%$ de los casos de PRR, respectivamente, ocurre, por lo general, en conjunción con la afección laríngea. ${ }^{2,3}$ La extensión al parénquima pulmonar se evidencia en menos del $1 \% .^{2}$ La PRR que se origina en la tráquea sin lesiones en la laringe es muy rara. ${ }^{4}$ Existen pocos casos documentados en la literatura mundial. ${ }^{1,5-8}$

Típicamente, los pacientes con papilomatosis laríngea se manifiestan con disfonía y estridor. La presentación clínica de la papilomatosis traqueobronquial es inespecífica. Los síntomas incluyen tos crónica, disnea, estridor bifásico, infecciones respiratorias recurrentes y/o hemoptisis. ${ }^{4-6,8}$ Se puede manifestar con síntomas de enfermedad reactiva de la vía aérea., ${ }^{5,6,8}$

Esta enfermedad tiene un curso clínico impredecible y tendencia a la recurrencia. Los tratamientos disponibles, tanto quirúrgicos como médicos, son solo paliativos, enfocados a mantener una vía aérea adecuada. ${ }^{3}$ Un gran desafío terapéutico lo constituyen las lesiones traqueobronquiales y pulmonares no accesibles a la intervención local. La inhibición del factor de crecimiento del endotelio vascular por el 
bevacizumab parece ser una opción nueva y eficaz. Si bien existen casos publicados en la literatura mundial del uso del bevacizumab sistémico para el tratamiento de la papilomatosis traqueobroncopulmonar en pediatría, no hay documentaciones previas en Argentina. ${ }^{9-11}$

Se presenta un caso muy raro de PRR con compromiso traqueobronquial y pulmonar sin afección laríngea, y se describe nuestra experiencia con el uso del bevacizumab sistémico.

FIgURA 1. Radiografía cervical simple de perfil. Disminución del calibre traqueal (flecha)

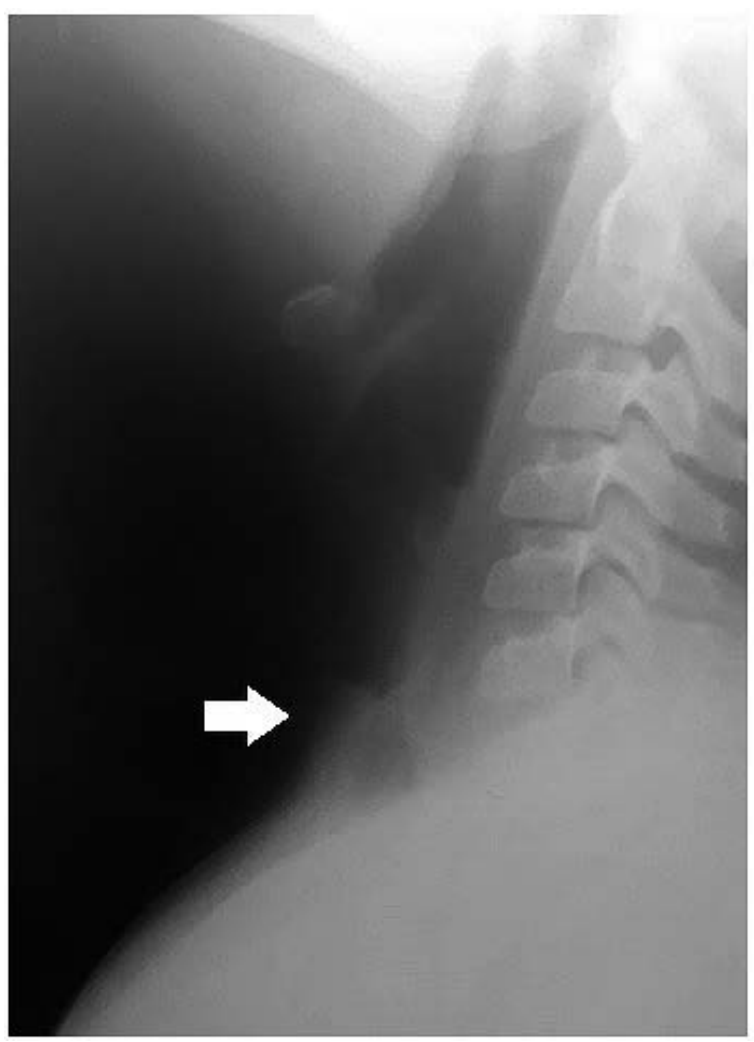

\section{CASO CLÍNICO}

Varón de 6 años con diagnóstico de asma que consultó a la Sección de Endoscopía Respiratoria por estridor bifásico y dificultad respiratoria moderada de 1 semana de evolución y tos crónica. Recibía tratamiento con inhalatorios beta agonistas y corticoides sistémicos sin mejoría de los síntomas respiratorios.

Como antecedentes, refirió neumonía recurrente izquierda e intubación endotraqueal con tubo con balón $n^{\circ} 5,5$ durante 8 días, debido a una crisis asmática 4 meses atrás. Nació por parto vaginal. No tenía historia previa de papilomatosis laríngea y la madre presentaba citología cervical negativa para VPH.

Con la sospecha de estenosis posintubación, se realizó una radiografía cervical de perfil, en la que se observó la disminución del calibre de la tráquea (Figura 1). Al efectuarse la evaluación endoscópica de la vía aérea con instrumental rígido, se evidenciaron múltiples lesiones de aspecto papilomatoso en toda la extensión de la tráquea y ambos bronquios fuentes, que ocluían la luz de la vía aérea en más del $90 \%$. La laringe era normal (Figura 2).

Se resecaron las lesiones con instrumental frío y se tomaron múltiples biopsias de diferentes sitios. El paciente presentó un alivio inmediato de los síntomas. La anatomía patológica confirmó el diagnóstico de papilomatosis con microfocos de displasia intensa. La tipificación viral fue positiva para el VPH-11.

Se realizó una tomografía computada (TC) de tórax y se observaron imágenes nodulares menores de $5 \mathrm{~mm}$, algunas de las cuales estaban cavitadas, dispersas en ambos pulmones, y bronquiectasias en el lóbulo inferior izquierdo y la língula. No se evidenciaron adenopatías (Figura 3).

Figura 2. Imágenes endoscópicas. A. Laringe normal. B y C. Papilomas en la tráquea proximal (B) y distal (C)

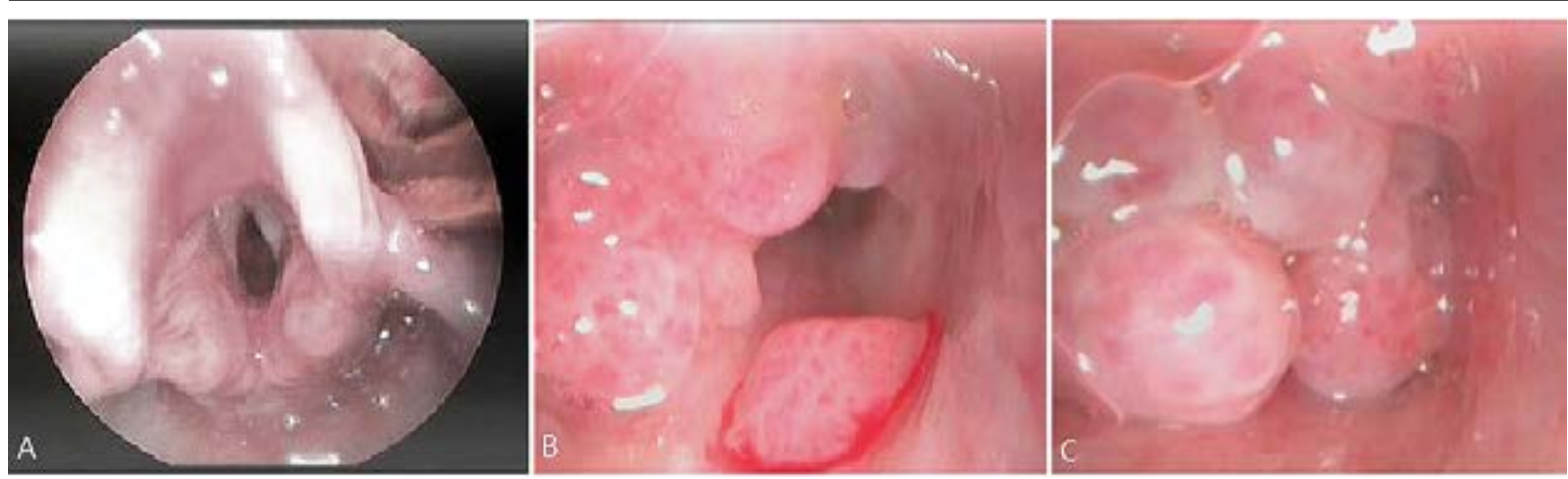


Al mes de la endoscopía, presentó recurrencia de los síntomas, por lo se realizó otra broncoscopía, en la que se evidenció el rápido crecimiento de las lesiones traqueales, las cuales se resecaron nuevamente.
Por la gravedad del compromiso traqueobronquial y pulmonar, se decidió iniciar un tratamiento con bevacizumab endovenoso (10 $\mathrm{mg} / \mathrm{kg}$ cada 4 semanas, 6 dosis).

FIGURA 3. A y B. Tomografía computada de tórax, cortes axiales. Se observan lesiones nodulares y cavitadas en el parénquima de ambos pulmones y bronquiectasias en el lóbulo inferior izquierdo y língula
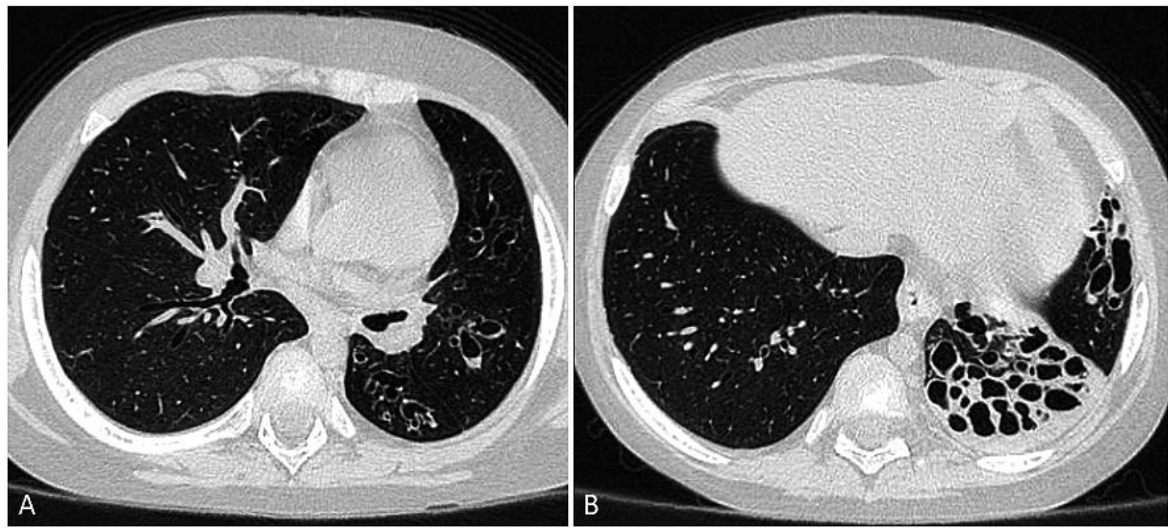

Figura 4. Endoscopía ( $A, B$ y C) y tomografía computada de tórax ( $D$ y E) posteriores al tratamiento sistémico. Tráquea proximal (A) y distal (B) y bronquios fuentes (C) sin lesiones exofíticas. D y E. Sin cambios significativos en el parénquima pulmonar respecto a la tomografía anterior
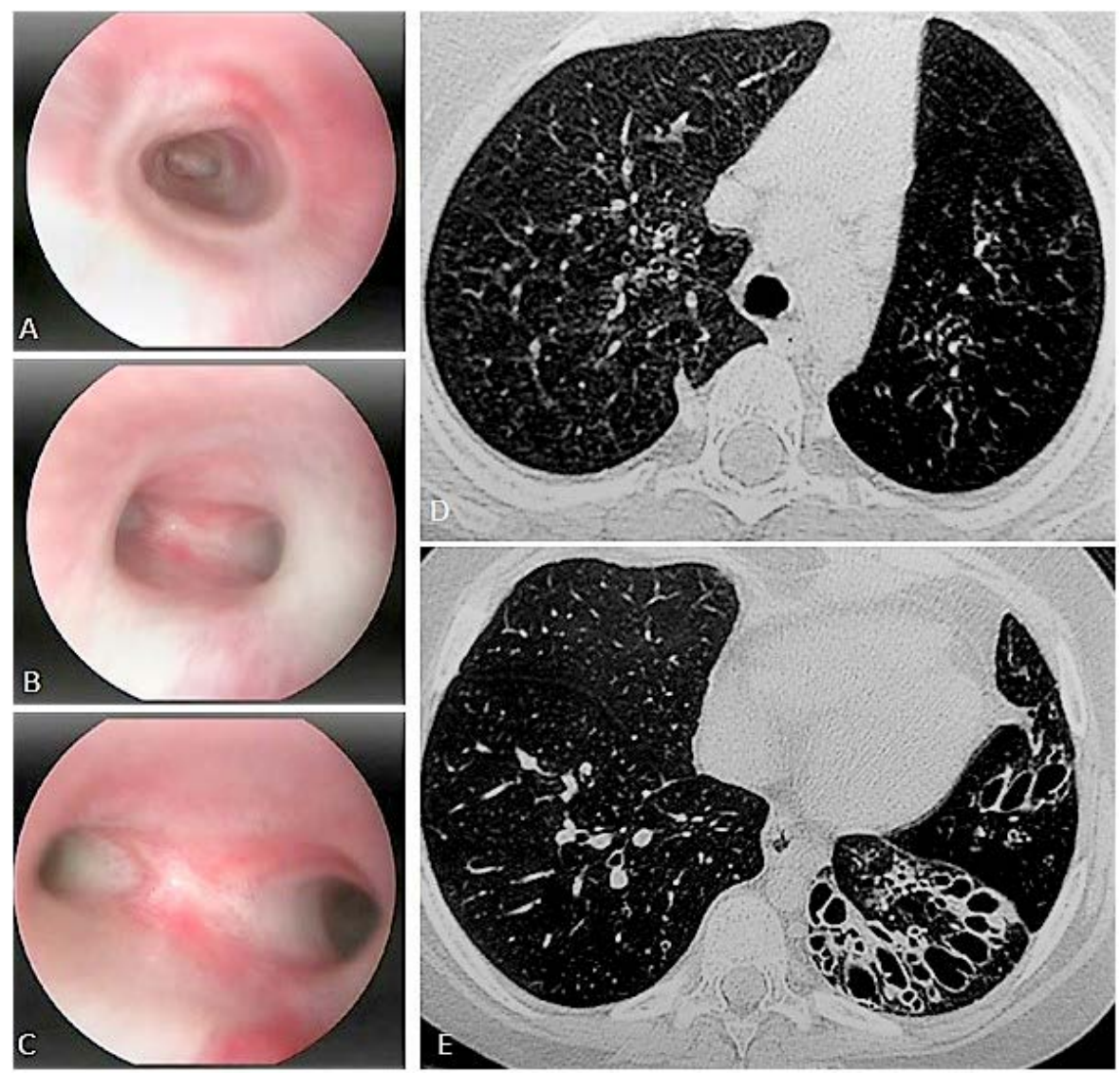
Se informó a los familiares de los riesgos de esta droga y su uso fuera de las indicaciones autorizadas. El Comité de Ética del Hospital aprobó el tratamiento.

El paciente se internó para la administración de cada dosis de la medicación. Se controló la presión arterial durante la infusión y se realizó un análisis de laboratorio antes y después de la infusión para evaluar la función renal y el perfil metabólico.

No hubo complicaciones. La broncoscopía al mes de haber iniciado el tratamiento sistémico y a los 6 meses reveló una notable mejoría de la enfermedad con escasos papilomas sésiles en la tráquea. La TC después de la sexta dosis no reveló cambios significativos en el parénquima pulmonar respecto a la anterior (Figura 4). El paciente no requirió nuevas intervenciones endoscópicas ni presentó progresión de la enfermedad. Continúa en seguimiento por Endoscopía. Se realizará una dosis de mantenimiento con bevacizumab endovenoso cada 3 meses. Se evaluará en forma periódica la eficacia y la seguridad de la medicación y el curso de la enfermedad.

\section{DISCUSIÓN}

La prevalencia de la PRR se estima de 1,45 a 2,93 cada 100000 niños. ${ }^{12}$ Las lesiones de la vía aérea inferior se deben, generalmente, a la diseminación de las lesiones laríngeas. ${ }^{1} \mathrm{El}$ compromiso traqueobronquial aislado que presentaba nuestro paciente es muy raro.

Si bien existen casos de transmisión del VPH después de una cesárea, el principal mecanismo de transmisión de la PRR juvenil es a través del canal de parto de una madre con infección vaginal latente o activa. ${ }^{5}$

La clínica de la papilomatosis traqueobronquial es insidiosa. Por lo general, se inicia con disnea de esfuerzo que progresa lentamente al reposo, y el estridor aparece cuando la obstrucción de la luz traqueal es mayor del $50 \%$. En ocasiones, se puede presentar con síntomas de hipersensibilidad bronquial. ${ }^{5,6,8}$ Los síntomas de nuestro paciente fueron tratados como asma en el inicio.

No es poco común la demora significativa hasta el diagnóstico apropiado. Diferenciar el asma de otras causas anatómicas de disnea y "sibilancias" puede ser un desafío. La obstrucción de la vía aérea se puede presentar con ruidos respiratorios clínicamente difíciles de distinguir de las sibilancias. ${ }^{13}$ La dificultad en el diagnóstico aumenta en situaciones en las que la obstrucción de la vía aérea se produce de forma insidiosa.

Los tumores traqueales, los tumores del mediastino, las anomalías de los grandes vasos que comprimen la vía aérea, el reflujo gastroesofágico y la aspiración de cuerpos extraños pueden mimetizar el asma. ${ }^{13}$

El rol de los estudios por imágenes en el diagnóstico diferencial es limitado. ${ }^{5}$ Las lesiones traqueales se pueden ver en la radiografía cervical, especialmente, en proyección lateral. La TC del tórax puede identificar lesiones en la tráquea, los bronquios y el parénquima pulmonar. ${ }^{4,5}$

La obstrucción fija o reversible en las pruebas de función respiratoria puede ayudar en el diagnóstico, pero no son siempre factibles de realizar en los niños. ${ }^{1,5}$

La endoscopía es fundamental para identificar las típicas lesiones vascularizadas de características verrugosas blanco-rosadas y evaluar la extensión de la enfermedad. Cuando hay compromiso traqueobronquial, se debe solicitar una TC de tórax. ${ }^{2,3}$ Las lesiones en el pulmón se presentan como nódulos que se cavitan con posterioridad. La anatomía patología confirma el diagnóstico.

La afectación pulmonar es más frecuente en el sexo masculino; se asocia con un curso más agresivo y con un bajo riesgo de transformación maligna (el 1-2 \%). ${ }^{2,14}$ No se conoce el mecanismo de diseminación, pero se sugiere la implantación periférica secundaria a manipulaciones de la vía aérea o bien un origen multicéntrico de la enfermedad. La realización de la traqueotomía facilita la diseminación. ${ }^{15}$

La presencia del VPH-11 en los papilomas se asocia a la enfermedad más agresiva. En los casos documentados de transformación maligna de las lesiones pulmonares, el VPH-11 fue el subtipo identificado., ${ }^{5,14} \mathrm{El}$ aumento de tamaño de una lesión previa y la presencia de adenopatías mediastínicas deben plantear la sospecha de malignidad. ${ }^{14}$ La PRR con diseminación intrapulmonar presagia un mal pronóstico.,10

Existen muchas modalidades de tratamiento para la PRR, pero ninguna de ellas ha demostrado ser completamente eficaz en todos los pacientes. La recurrencia es frecuente porque el VPH puede persistir de manera latente en el tejido normal adyacente a las lesiones. ${ }^{1}$

La remoción quirúrgica endoscópica (con instrumental frío, láser o microdebridador) sigue siendo el tratamiento fundamental para asegurar una vía aérea adecuada. ${ }^{1,5}$ 
Las indicaciones actuales para el tratamiento médico adyuvante son la necesidad de más de 4 intervenciones por año, la diseminación de la enfermedad y el rápido crecimiento de la lesión que cause la obstrucción de la vía aérea. ${ }^{5}$

El tratamiento adyuvante incluye terapias locales y sistémicas, tales como antivirales (por ejemplo, cidofovir), interferón, bevacizumab y vacuna contra el VPH. ${ }^{4}$

El bevacizumab es un anticuerpo monoclonal contra el factor de crecimiento endotelial vascular, que funcionaría como un inhibidor de la angiogénesis y obstaculizaría el crecimiento de papilomas. ${ }^{10-12,14} \mathrm{Su}$ administración sistémica se presenta como una opción de tratamiento efectiva en la PRR con lesiones bronquiales y pulmonares no accesibles al tratamiento local. ${ }^{9,10}$

El bevacizumab es ampliamente utilizado en el tratamiento del cáncer metastásico. Se ha documentado que es bien tolerado, pero, a veces, se asocia con reacciones adversas graves. Los efectos adversos más frecuentes de esta droga son la hipertensión arterial, la proteinuria y la trombosis. ${ }^{11}$ Algunos informes indican que el uso del bevacizumab en los niños parece ser seguro, con efectos adversos que son reversibles con la interrupción del tratamiento. ${ }^{11}$ Se sugiere medir de forma rutinaria la presión arterial durante el tratamiento y después de él por 4-6 meses. ${ }^{11}$

La PRR sin compromiso de la laringe es muy rara. Su diagnóstico requiere un alto grado de sospecha clínica. En todo niño que no responde a la terapia inicial de asma, se deben considerar otras causas de disnea y "sibilancias"; la endoscopía y la TC son de gran utilidad en el diagnóstico. El bevacizumab sistémico podría representar una estrategia prometedora en el tratamiento de la PRR con compromiso traqueal y broncopulmonar. ${ }^{3,11}$ Es importante el seguimiento del paciente, debido a la tendencia a la recurrencia y a la posibilidad de transformación maligna, y para monitorizar la aparición de posibles efectos adversos del tratamiento a largo plazo. ${ }^{5}$

\section{REFERENCIAS}

1. Xue Q, Wang J. Recurrent respiratory papillomatosis arising in trachea not affecting larynx. Intern Med. 2010; 49(15):1649-51.

2. Monnier P. Recurrent respiratory papillomatosis. En: Monnier P (ed.). Pediatric airway surgery. Management of laryngotracheal stenosis in infants and children. Berlin/ Heidelberg: Springer; 2011.Págs.220-7.

3. Rodríguez H, Cuestas G, Álvarez M, Monaje M. Papilomatosis respiratoria recurrente con compromiso pulmonar y de la pared torácica: una rara complicación en un adolescente. Acta Otorrinolaringol Esp. 2017; 69(2):110-3.

4. Kalanjeri S, Hoffman S, Farver C, Almeida F. Diffuse tracheal papillomatosis. Am J Respir Crit Care Med. 2017; 195(1):134-5.

5. Yildirim F, Türk M, Demircan S, Akürek N, et al. Tracheal papilloma treated with cryotherapy and interferon- $\alpha$ : A case report and review of the literature. Case Rep Pulmonol. 2015; 2015:356796.

6. Chen Y, Jiang J, Guo L, Huang J. Primary tracheal papilloma disguised as asthma: A case report. J Asthma. 2016; 53(10):1090-3.

7. Breen D, Lyons O, Barrett H, Burke C. Isolated tracheal papillomatosis-An infrequent cause of chronic cough. Respir Med Extra. 2007; 3(1):21-2.

8. ValentinoJ, BrameCB, Studtmann KE, Manaligod J.Primary tracheal papillomatosis presenting as reactive airway disease. Otolaryngol Head Neck Surg. 2002; 126(1):79-80.

9. Bedoya A, Glisinki K, Clarke J, Lind R, et al. Systemic bevacizumab for recurrent respiratory papillomatosis: A single center experience of two cases. Am J Case Rep. 2017; 18:842-6.

10. Mohr M, Schliemann C, Biermann C, Schmidt L, et al. Rapid response to systemic bevacizumab therapy in recurrent respiratory papillomatosis. Oncol Lett. 2014; 8(5):1912-8.

11. ZurKB, FoxE. Bevacizumab chemotherapy for management of pulmonary and laryngotracheal papillomatosis in a child. Laryngoscope. 2017; 127(7):1538-42.

12. Marsico M, Mehta V, Chastek B, Liaw K, et al. Estimating the incidence and prevalence of juvenile onset recurrent respiratory papillomatosis in publicly and privately insured claims databases in the United States. Sex Transm Dis. 2014; 41(5):300-5.

13. Nunn AC, Nouraei SAR, George JP, Sandhu G, et al. Not always asthma: Clinical and legal consequences of delayed diagnosis of laryngotracheal stenosis. Case Rep Otolaryngol. 2014; 2014:325048.

14. Xiao Y, Wang J, Han D, Ma L. A case of the intrapulmonary spread of recurrent respiratory papillomatosis with malignant transformation. Am J Med Sci. 2015; 350(1):55-7.

15. Wang J, Han DM, Ma LJ, Ye JY, et al. Risk factors of juvenile onset recurrent respiratory papillomatosis in the lower respiratory tract. Chin Med J (Engl). 2012; 125(19):3496-9. 